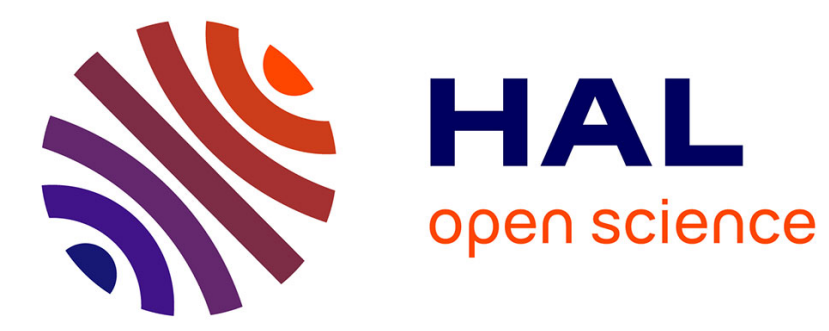

\title{
Dynamics of coupled oscillators excited by dry friction
} Madeleine Pascal

\section{To cite this version:}

Madeleine Pascal. Dynamics of coupled oscillators excited by dry friction. Journal of Computational and Nonlinear Dynamics, 2008, 3 (3), pp.20-26. 10.1115/1.2908272 . hal-00783548

\section{HAL Id: hal-00783548 \\ https://hal.science/hal-00783548}

Submitted on 6 Jun 2016

HAL is a multi-disciplinary open access archive for the deposit and dissemination of scientific research documents, whether they are published or not. The documents may come from teaching and research institutions in France or abroad, or from public or private research centers.
L'archive ouverte pluridisciplinaire HAL, est destinée au dépôt et à la diffusion de documents scientifiques de niveau recherche, publiés ou non, émanant des établissements d'enseignement et de recherche français ou étrangers, des laboratoires publics ou privés. 


\title{
Dynamics of Coupled Oscillators Excited by Dry Friction
}

\author{
Madeleine Pascal
}

In this paper, we present an analytical method to investigate the behavior of a two-degree-of-freedom oscillator excited by dry friction. The system consists of two masses connected by linear springs. These two masses are in contact with a driving belt moving at a constant velocity. The contact forces between the masses and the belt are obtained assuming Coulomb's friction law. Two families of periodic motions are found in closed form. The first one includes stick-slip oscillations with two switches per period, the second one is also composed of stick-slip motion, but includes three switches per period. In both cases, the initial conditions and the time duration of each kind of motions (stick or slip phases) are obtained in analytical form.

Keywords: coupled oscillators, dry friction, periodic motions, analytical method, stick-slip motion

\section{Introduction}

Vibrating systems excited by dry friction are frequently encountered in technical applications, including turbine blade joints, robot joints, electric motor drives, wheel rail coupling of mass transit systems, and brake systems. These systems are strongly nonlinear and they are usually modeled as spring-mass oscillators. They have been the subject of several investigations $[1,2]$. In the simplest case of a one-degree-of-freedom system [3], an analytical investigation of the system's behavior, including periodic motions, chatter, and chaos, have been performed. In the case of multidegrees-of-freedom systems [4], only the numerical approaches have been used. In this work, our attention is focused on the analysis of an undamped self-excited two-degree-of-freedom oscillator with dry friction. We assume Coulomb's friction law and, by using the method already presented in Ref. [5] for a coupled oscillator with impact, periodic motions with stick-slip oscillations are obtained by analytical methods.

\section{Model}

The system (Fig. 1) consists of two masses $m_{1}, m_{2}$, connected by linear springs $k_{1}, k_{2}$. These two masses are in contact with a driving belt moving at a constant velocity $v_{0}$. Friction forces $F_{1}, F_{2}$ act between the masses $m_{1}, m_{2}$ and the belt.

This two-degree-of-freedom oscillator is governed by the following differential system:

$$
\begin{gathered}
M \ddot{X}+K X=R, \quad X=\left(x_{1}, x_{2}\right)^{t}, \quad R=\left(F_{1}, F_{2}\right)^{t} \\
M=\left(\begin{array}{cc}
m_{1} & 0 \\
0 & m_{2}
\end{array}\right), \quad K=\left(\begin{array}{cc}
k_{1}+k_{2} & -k_{2} \\
-k_{2} & k_{2}
\end{array}\right) \\
X=X\left(t^{\prime}\right), \quad \ddot{X}=\frac{d^{2} X}{d t^{\prime 2}}
\end{gathered}
$$

Here, $F_{1}, F_{2}$ are the contact friction forces obtained from Coulomb's laws:

$$
\begin{gathered}
\nu_{0}-\dot{x}_{i} \neq 0, \quad F_{i}=F_{s i} \operatorname{sign}\left(\nu_{0}-\dot{x}_{i}\right) \quad(i=1,2) \\
\nu_{0}-\dot{x}_{1}=0
\end{gathered}
$$

$$
\begin{gathered}
F_{1} \\
= \begin{cases}\left(k_{1}+k_{2}\right) x_{1}-k_{2} x_{2} & \text { if }\left|\left(k_{1}+k_{2}\right) x_{1}-k_{2} x_{2}\right|<F_{r 1} \\
\varepsilon F_{s 1} & \text { if } \varepsilon\left[\left(k_{1}+k_{2}\right) x_{1}-k_{2} x_{2}\right]>F_{r 1}(\varepsilon= \pm 1) \\
\nu_{0}-\dot{x}_{2}=0\end{cases} \\
F_{2}= \begin{cases}k_{2}\left(x_{2}-x_{1}\right) & \text { if }\left|k_{2}\left(x_{2}-x_{1}\right)\right|<F_{r 2} \\
\varepsilon F_{s 2} & \text { if } \varepsilon k_{2}\left(x_{2}-x_{1}\right)>F_{r 2}(\varepsilon= \pm 1)\end{cases}
\end{gathered}
$$

$F_{s 1}, F_{s 2}$ are the friction forces when slip motion occurs, while $F_{r 1}, F_{r 2}$ are the static friction forces. $\left(F_{s i}<F_{r i}\right)$

The systems (1) and (2) are normalized using

$$
t=\omega_{3} t^{\prime}, \quad \omega_{3}=\sqrt{\frac{k_{1}+k_{2}}{m_{1}}}, \quad\left(o^{\prime}\right)=\frac{d(o)}{d t}
$$

From Eq. (1), it follows

$$
\begin{gathered}
x_{1}^{\prime \prime}+x_{1}-\chi x_{2}=u_{1}, \quad \chi=\frac{k_{2}}{k_{1}+k_{2}}, \quad \eta=\frac{m_{1}}{m_{2}} \\
x_{2}^{\prime \prime}+\chi \eta\left(x_{2}-x_{1}\right)=\eta u_{2}, \quad u_{i}=\frac{F_{i}}{k_{1}+k_{2}} \quad(i=1,2)
\end{gathered}
$$

\section{Prediction of the Oscillations Exhibited by the Sys- tem}

The coupled oscillator system excited by dry friction is modeled as a piecewise-linear system. The dynamical behavior is very complex and includes slip or stick oscillations of the masses. All possible motions of the system are composed of several phases of slip motion for both masses, stick motion of $m_{1}$, and slip motion of $m_{2}$ or vice versa and at last, stick motion for the two masses. For each kind of motions, the close form solution can be obtained.

3.1 Slip Motion of the Two Masses. This motion is related to the following initial conditions: $x_{i 0}, x_{i 0}^{\prime}<V(i=1,2),\left(V=\nu_{0} / \omega_{3}\right)$. The motion of the oscillators is given by the solution of Eq. (3) with $u_{i}=u_{s i}(i=1,2)$ :

$$
\begin{gathered}
Z^{+}(t)=H(t) Z_{0}^{+}, \quad Z^{+}=\left(\begin{array}{c}
X-d_{0} \\
X^{\prime}
\end{array}\right), \quad Z_{0}^{+}=Z^{+}(0), \quad X=\left(x_{1}, x_{2}\right)^{t} \\
H=\left(\begin{array}{c}
H_{1} H_{2} \\
H_{3} H_{1}
\end{array}\right), \quad H_{i}=\Lambda B_{i} \Lambda^{-1} \quad(i=1,2,3)
\end{gathered}
$$




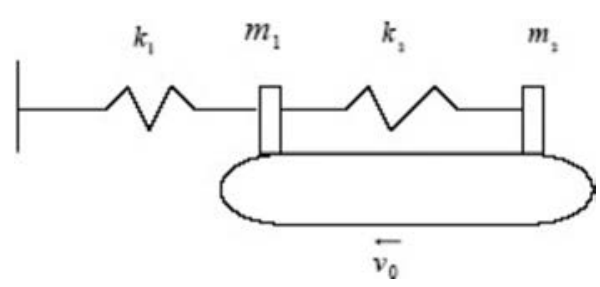

Fig. 1 Coupled oscillators with dry friction

$$
\begin{gathered}
\Lambda=\left(\begin{array}{cc}
1 & 1 \\
\lambda_{1} & \lambda_{2}
\end{array}\right), \quad B_{1}=\left(\begin{array}{cc}
c_{1} & 0 \\
0 & c_{2}
\end{array}\right), \quad B_{2}=\left(\begin{array}{cc}
\frac{s_{1}}{\omega_{1}} & 0 \\
0 & \frac{s_{2}}{\omega}
\end{array}\right) \\
B_{3}=B_{1}^{\prime}, \quad c_{i}=\cos \left(\omega_{i} t\right), \quad s_{i}=\sin \left(\omega_{i} t\right) \quad(i=1,2)
\end{gathered}
$$

where $\omega_{1}, \omega_{2}$ are the roots of the characteristic equation:

$$
\begin{gathered}
\operatorname{det}\left(\tilde{K}-I \omega^{2}\right)=0, \\
\tilde{K}=\left(\begin{array}{cc}
1 & -\chi \\
-\chi \eta & \chi \eta
\end{array}\right), \quad I=\left(\begin{array}{ll}
1 & 0 \\
0 & 1
\end{array}\right)
\end{gathered}
$$

$\psi_{i}=\left(\begin{array}{c}1 \\ \lambda_{i}\end{array}\right)(i=1,2)$ are defined by $\left(\tilde{K}-I \omega_{i}^{2}\right) \psi_{i}=0$.

At last, $d_{0}=\left(d_{01}, d_{02}\right)^{t}$ is the constant part of the solution

$$
d_{01}=\frac{u_{s 1}+u_{s 2}}{1-\chi}, \quad d_{02}=\frac{u_{s 1}}{1-\chi}+\frac{u_{s 2}}{\chi(1-\chi)}
$$

The $H_{i}$ matrices fulfill the following properties [5]:

$$
\begin{gathered}
H_{1}^{2}-H_{2} H_{3}=I \\
H_{i} H_{j}=H_{j} H_{i} \quad(i, j=1,2,3)
\end{gathered}
$$

Several variants of this slip-slip motion exist:

$$
Z^{-}(t)=H(t) Z_{0}^{-}, \quad Z^{-}=\left(\begin{array}{c}
X+d_{0} \\
X^{\prime}
\end{array}\right)
$$

related to initial conditions $x_{i 0}, x_{i 0}^{\prime}>V(i=1,2)$.

$$
\begin{gathered}
Y^{+}(t)=H(t) Y_{0}^{+}, \quad Y^{+}=\left(\begin{array}{c}
X-\bar{d}_{0} \\
X^{\prime}
\end{array}\right), \quad \bar{d}_{0}=\left(\bar{d}_{01}, \bar{d}_{02}\right)^{t} \\
\bar{d}_{01}=\frac{u_{s 1}-u_{s 2}}{1-\chi}, \quad \bar{d}_{02}=\frac{u_{s 1}}{1-\chi}-\frac{u_{s 2}}{\chi(1-\chi)}
\end{gathered}
$$

related to initial conditions $x_{10}^{\prime}<V, x_{20}^{\prime}>V$.

$$
Y^{-}(t)=H(t) Y_{0}^{-}, \quad Y^{-}=\left(\begin{array}{c}
X+\bar{d}_{0} \\
X^{\prime}
\end{array}\right)
$$

related to initial conditions $x_{10}^{\prime}>V, x_{20}<V$.

3.2 Slip Motion of the First Mass and Stick Motion of the Second Mass. This motion is related to the following initial conditions:

$$
x_{10}^{\prime}<V, \quad x_{20}^{\prime}=V, \quad \chi\left|x_{20}-x_{10}\right|<u_{r 2}
$$

The motion equations are given by

$$
x_{1}^{\prime \prime}+x_{1}-\chi x_{2}=u_{s 1}, \quad x_{2}^{\prime \prime}=0
$$

The solution of this linear system is obtained in analytical form:

$$
\begin{gathered}
\bar{Z}^{+}(t)=\Gamma(t) \bar{Z}_{0}^{+}, \quad \bar{Z}^{+}=\left(\begin{array}{c}
X-\delta_{0} \\
X^{\prime}
\end{array}\right), \quad \bar{Z}_{0}^{+}=\bar{Z}^{+}(0) \\
\Gamma=\left(\begin{array}{cc}
\Gamma_{1} & \Gamma_{2} \\
\Gamma_{3} & \Gamma_{1}
\end{array}\right), \quad \Gamma_{i}=\Sigma \gamma_{i} \Sigma^{-1} \quad(i=1,2,3)
\end{gathered}
$$

$$
\begin{gathered}
\Sigma=\left(\begin{array}{ll}
1 & \chi \\
0 & 1
\end{array}\right), \quad \gamma_{1}=\left(\begin{array}{cc}
\cos t & 0 \\
0 & 1
\end{array}\right), \quad \gamma_{2}=\left(\begin{array}{cc}
\sin t & 0 \\
0 t &
\end{array}\right), \\
\gamma_{3}=\gamma_{1}^{\prime}, \quad \delta_{0}=\left(u_{s 1}, 0\right)^{t}
\end{gathered}
$$

For the $\Gamma_{i}$ matrices, the following properties hold:

$$
\Gamma_{1}^{2}-\Gamma_{2} \Gamma_{3}=I, \quad \Gamma_{i} \Gamma_{j}=\Gamma_{j} \Gamma_{i} \quad(i, j=1,2,3)
$$

$$
\left(\Gamma_{1}-I\right)\left(\delta_{0}-d_{0}\right)=0, \quad \Gamma_{3}\left(\delta_{0}-d_{0}\right)=0
$$

The solution can be also formulated as

$$
Z^{+}(t)=\Gamma(t) Z_{0}^{+}, \quad Z^{+}=\left(\begin{array}{c}
X-d_{0} \\
X^{\prime}
\end{array}\right)
$$

One variant of this solution is related to the initial conditions:

$$
x_{10}^{\prime}>V, \quad x_{20}^{\prime}=V, \quad \chi\left|x_{20}-x_{10}\right|<u_{r 2}
$$

3.3 Stick Motion of the First Mass, Slip Motion of the Second Mass. This motion is related to the following initial conditions:

$$
x_{10}^{\prime}=V, \quad x_{20}^{\prime}<V, \quad\left|x_{10}-\chi x_{20}\right|<u_{r 1}
$$

and to the following motion equations:

$$
x_{1}^{\prime \prime}=0
$$

$$
x_{2}^{\prime \prime}+\chi \eta\left(x_{2}-x_{1}\right)=\eta u_{s 2}
$$

The solution is given by

$$
\begin{gathered}
\tilde{Z}^{+}(t)=C(t) \tilde{Z}_{0}^{+}, \quad \tilde{Z}^{+}=\left(\begin{array}{l}
X-e_{0} \\
X^{\prime}
\end{array}\right), \quad \tilde{Z}_{0}^{+}=\tilde{Z}^{+}(0), \quad C=\left(\begin{array}{ll}
C_{1} & C_{2} \\
C_{3} & C_{1}
\end{array}\right) \\
C_{i}=\Pi A_{i} \Pi^{-1} \quad(i=1,2,3), \quad \Pi=\left(\begin{array}{ll}
1 & 0 \\
1 & 1
\end{array}\right) \\
A_{1}=\left(\begin{array}{cc}
1 & 0 \\
0 & \cos (\omega t)
\end{array}\right), \quad A_{2}=\left(\begin{array}{cc}
t & 0 \\
0 & \frac{\sin (\omega t)}{\omega}
\end{array}\right), \quad A_{3}=A_{1}^{\prime} \\
e_{0}=\left(0, \frac{u_{s 2}}{\omega}\right)^{t}, \quad \omega^{2}=\chi \eta
\end{gathered}
$$

As for the $\Gamma_{i}$ matrices, the following properties hold for the $C_{i}$ matrices:

$$
\begin{gathered}
C_{1}^{2}-C_{2} C_{3}=I \\
C_{i} C_{j}=C_{i} C_{j} \quad(i, j=1,2,3) \\
\left(C_{1}-I\right)\left(e_{0}-d_{0}\right)=0, \quad C_{3}\left(e_{0}-d_{0}\right)=0
\end{gathered}
$$

Eq. (18) can be formulated as

$$
Z^{+}(t)=C(t) Z_{0}^{+}, \quad Z^{+}=\left(\begin{array}{c}
X-d_{0} \\
X^{\prime}
\end{array}\right)
$$

One variant of this solution is related to the initial conditions

$$
x_{10}^{\prime}=V, \quad x_{20}^{\prime}>V, \quad\left|x_{10}-\chi x_{20}\right|<u_{r 1}
$$

3.4 Stick Motion of the Two Masses. This last case is related to the initial conditions:

$$
x_{10}^{\prime}=x_{20}^{\prime}=V, \quad\left|x_{10}-\chi x_{20}\right|<u_{r 1}, \quad \chi\left|x_{20}-x_{10}\right|<u_{r 2}
$$

The two masses perform a stick motion given by:

$$
X=X_{0}+t X_{0}^{\prime}, \quad X^{\prime}=X_{0}^{\prime}
$$




\section{Periodic Motions With Transitions Between Slip and Stick Motions}

A transition between slip and stick motions occurs if at least one of the velocities of the masses reaches the velocity of the belt. In this case, the eventual transition depends on some criteria related to the restoring force applied to the mass (i.e., on Coulomb's laws (2)). A detailed description of the analytical predictions of the stick and nonstick motions for a one-degree-of-freedom damped and forced oscillator with dry friction can be found in Ref. [6]. In the following, periodic solutions including two or three transitions between each kind of motions are obtained in analytical form.

4.1 Solution I: Periodic Motion With Two Transitions per Period. Let us assume that at $t=0$,

$$
x_{10}^{\prime}<V, \quad x_{20}^{\prime}=V, \quad \chi\left(x_{20}-x_{10}\right)=u_{r 2}
$$

From Eq. (3), we deduce $x_{2}^{\prime \prime}(0)=\eta\left(u_{2}-u_{r 2}\right)$.

A motion with $u_{2}=u_{s 2}$ gives $x_{2}^{\prime \prime}(0)<0$; hence for $t>0, x_{2}^{\prime}$ is a decreasing function of $t$, and for some period of time $(0<t<\tau)$ $x_{2}^{\prime}<V$. The motion of the oscillators is given by Eq. (4).

This motion finishes at time $t=\tau$, if $x_{2 c}^{\prime}=x_{2}^{\prime}(\tau)=V$. Let us assume that $X_{c}=X(\tau), X_{c}^{\prime}=X^{\prime}(\tau)$ are given by

$$
X_{c}-d_{0}=-\left(X_{0}-d_{0}\right), \quad X_{c}^{\prime}=X_{0}^{\prime}
$$

From Eq. (4), it follows that $X_{0}, X_{0}^{\prime}$ are deduced from the conditions:

$$
\begin{gathered}
\left(H_{1}+I\right)\left(X_{0}-d_{0}\right)+H_{2} X_{0}^{\prime}=0 \\
H_{3}\left(X_{0}-d_{0}\right)+\left(H_{1}-I\right) X_{0}^{\prime}=0 \\
H_{i}=H_{i}(\tau) \quad(i=1,2,3)
\end{gathered}
$$

This system provides four scalar equations for the determination of the three unknown parameters $x_{10}, x_{10}^{\prime}, \tau\left(x_{20}, x_{20}^{\prime}\right.$ are deduced from conditions (22)). Taking into account the properties (8) of the $H_{i}$ matrices, these equations are not independent and reduce only to two scalar equations. From Eq. (23), we deduce

$$
\chi\left(x_{2 c}-x_{1 c}\right)=-\chi\left(x_{20}-x_{10}\right)+2 \chi\left(d_{02}-d_{01}\right)=-u_{r 2}+2 u_{s 2}<u_{r 2}
$$

It results that for $\left.t>\tau, \chi \mid x_{2}-x_{1}\right) \mid<u_{r 2}$, and the first mass performs a slip motion while the second one performs a stick motion. From Eq. (16), the solution is written in the following form:

$$
Z^{+}(t)=\Gamma(t-\tau) Z_{c}^{+}, \quad Z_{c}^{+}=E Z_{0}^{+}, \quad E=\left(\begin{array}{cc}
-I & 0 \\
0 & I
\end{array}\right)
$$

This motion ends at $t=\tau+T$, when $\chi\left(x_{2}-x_{1}\right)$ reaches the limiting value $u_{r 2}$. This condition is fulfilled if we assume that $x_{i}(\tau+T)$ $=x_{i 0}, x_{i}^{\prime}(\tau+T)=x_{i 0}^{\prime},(i=1,2)$.

It results to the following conditions:

$$
\begin{gathered}
\left(\Gamma_{1}+I\right)\left(X_{0}-d_{0}\right)-\Gamma_{2} X_{0}^{\prime}=0 \\
-\Gamma_{3}\left(X_{0}-d_{0}\right)+\left(\Gamma_{1}-I\right) X_{0}^{\prime}=0 \\
\Gamma_{i}=\Gamma_{i}(T) \quad(i=1,2,3)
\end{gathered}
$$

Taking into account the properties (15) of the $\Gamma_{i}$ matrices, these four scalar equations are not independent and reduce only to two scalar conditions. It results that Eqs. (24) and (27) provide four scalar equations for the determination of the four parameters $x_{10}, x_{10}^{\prime}, \tau, T$. To each solution of these equations, a periodic motion of the coupled oscillators of period $\tau+T$, with two transitions per period, is obtained.

In conclusion, the initial conditions and the duration times of the slip motion $\tau$ and of the stick motion $T$ related to a periodic solution (with one slip-slip mode and one slip-stick mode per period) are obtained from the system

$$
\begin{gathered}
x_{20}=\frac{V T}{2}+d_{02}, \quad x_{10}=x_{20}-\frac{u_{r 2}}{\chi} \\
x_{10}^{\prime}=\chi V+\cot \left(\frac{T}{2}\right)\left[\frac{u_{s 2}-u_{r 2}}{\chi}+\frac{V T}{2}(1-\chi)\right] \\
\left(H_{11}+1\right)\left(\frac{u_{s 2}-u_{r 2}}{\chi}+\frac{V T}{2}\right)+H_{12}\left(\frac{V T}{2}\right)+H_{13} x_{10}^{\prime}+H_{14} V=0 \\
H_{21}\left(\frac{u_{s 2}-u_{r 2}}{\chi}+\frac{V T}{2}\right)+\left(H_{22}+1\right)\left(\frac{V T}{2}\right)+H_{23} x_{10}^{\prime}+H_{24} V=0 \\
H_{1}=\left(H_{i j}\right) \quad(i, j=1,2), \quad H_{2}=\left(H_{i j}\right) \quad(i=1,2 ; j=3,4)
\end{gathered}
$$

However, to obtain a realistic solution, the results deduced from system (28) must fulfill the conditions

$$
\begin{gathered}
\tau>0, \quad T>0, \quad x_{1}^{\prime}-V<0, \quad x_{2}^{\prime}-V<0 \quad(0<t<\tau) \\
z_{1} \equiv \chi\left(x_{2}-x_{1}\right)-u_{r 2}<0 \\
z_{2} \equiv \chi\left(x_{2}-x_{1}\right)+u_{r 2}>0 \quad(\tau<t<\tau+T)
\end{gathered}
$$

An interesting property of symmetry can be found for this solution (see Appendix and Ref. [7]):

$$
\begin{gathered}
Z(t)=E Z(\tau-t), \quad 0<t<\tau / 2 \\
Z(t)=E Z(2 \tau+T-t), \quad \tau<t<\tau+T / 2
\end{gathered}
$$

It seems that a similar periodic solution without this property cannot be found for any set of data. It is not difficult to show that dissymmetrical periodic solutions such as Solution I can be found only for some particular values of the data.

\subsection{Solution II: Periodic Solution With Three Transitions} per Period. Let us assume that at $t=0$,

$$
x_{10}^{\prime}=x_{20}^{\prime}=V, \quad x_{10}-\chi x_{20}=u_{r 1}, \quad \chi\left|x_{20}-x_{10}\right|<u_{r 2}
$$

For some period of time $(0<t<\tau)$, the motion is composed of a slip motion for $m_{1}\left(u_{1}=u_{s 1}, x_{1}^{\prime}<V\right)$ and a stick motion for $m_{2}$ :

$$
Z^{+}(t)=\Gamma(t) Z_{0}^{+}
$$

A transition occurs at $t=\tau$ if

$$
\chi\left(x_{2 c}-x_{1 c}\right)=u_{r 2}, \quad x_{i c}=x_{i}(\tau), \quad(i=1,2)
$$

For a new period of time $\left(\tau<t<\tau+\tau_{1}\right)$, the the two masses undergo a slip motion:

$$
Z^{+}(t)=H(t-\tau) Z_{c}^{+}, \quad Z_{c}^{+}=Z^{+}(\tau)
$$

Again, a switch occurs at $t=\tau+\tau_{1}$ if

$$
\begin{gathered}
x_{1 d}^{\prime}=x_{1}^{\prime}\left(\tau+\tau_{1}\right)=V, \quad\left|x_{1 d}-\chi x_{2 d}\right|<u_{r 1}, \quad x_{i d}=x_{i}\left(\tau+\tau_{1}\right) \\
(i=1,2)
\end{gathered}
$$

For $\tau+\tau_{1}<t<\tau+\tau_{1}+T$, the first mass undergoes a stick motion, while the second one undergoes a slip motion:

$$
Z^{+}(t)=C\left(t-\tau-\tau_{1}\right) Z_{d}^{+}, \quad Z_{d}^{+}=Z^{+}\left(\tau+\tau_{1}\right)
$$

A periodic solution of period $\tau+\tau_{1}+T$ is obtained under the conditions

$$
Z^{+}\left(\tau+\tau_{1}+T\right) \equiv C(T) Z_{d}^{+}=Z_{0}^{+}
$$

This condition includes the relation $x_{1 d}^{\prime}=V$.

The obtained periodic solution depends on the four parameters: $x_{20}$ (the other initial conditions are obtained from Eq. (30)), and 
$\tau, \tau_{1}, T$ (time duration of each part of the periodic solution). On the other hand, the conditions (32) and (36) provide five scalar equations for the determination of these parameters. It results that in contrast to the periodic Solution I, this new solution is obtained only for some particular set of the data, which includes $\chi, \eta, V, u_{s i}, u_{r i},(i=1,2)$. The periodicity condition (36) gives the following equations:

$$
\begin{gathered}
N_{1}\left(X_{0}-d_{0}\right)+N_{2} X_{0}^{\prime}=0 \\
N_{3}\left(X_{0}-d_{0}\right)+N_{4} X_{0}^{\prime}=0 \\
N=C(T) H\left(\tau_{1}\right) \Gamma(\tau)-I_{4}=\left(\begin{array}{ll}
N_{1} & N_{2} \\
N_{3} & N_{4}
\end{array}\right) \\
I_{4}=\left(\begin{array}{ll}
I & 0 \\
0 & I
\end{array}\right), \quad X_{0}^{\prime}=\left(\begin{array}{l}
V \\
V
\end{array}\right)
\end{gathered}
$$

From Eq. (37), we deduce two compatibility conditions:

$$
\begin{gathered}
M_{11}+M_{12}=0, \quad M_{21}+M_{22}=0 \\
M \equiv\left(\begin{array}{l}
M_{11} M_{12} \\
M_{21} M_{22}
\end{array}\right)=N_{1}^{-1} N_{2}-N_{3}^{-1} N_{4}
\end{gathered}
$$

The relations (38) provide two equations linking the time duration $\left(\tau, \tau_{1}, T\right)$ of each part of the motion. From Eq. (37), we deduce the value of $X_{0}-d_{0}$ :

$$
X_{0}-d_{0}=-N_{1}^{-1} N_{2} X_{0}^{\prime}
$$

This last result, together with Eq. (38), gives four scalar equations for the determination of the parameters $x_{20}, \tau, \tau_{1}, T$ in terms of the data. Inserting these values in the condition (32), a compatibility condition for the existence of such a periodic solution is obtained. This compatibility condition provides, for example, the value of $u_{r 2}$, in terms of the data $\left(\chi, \eta, V, u_{r 1}, u_{s i},(i=1,2)\right)$.

\section{Numerical Applications}

Numerical validations will be made by using the MATLAB program for several values of the data $\chi, \eta, V, u_{r 1}, u_{s i}(i=1,2)$ and for each kind of periodic motions.

Solution I. From Eq. (28), the time duration $\tau$ of the slip motion is obtained from the condition

$$
\begin{gathered}
F(\tau) \equiv a_{11}+a_{12}-\chi\left(a_{21}+a_{22}\right)+B \varphi_{1} \varphi_{2} \\
+\left[1-\chi+B\left(a_{22}+\chi a_{21}\right)\right] \cot \left(\frac{B a_{21}-1}{a_{21}+a_{22}}\right) \\
=0 \\
a_{11}=d\left(\lambda_{1} \varphi_{2}-\lambda_{2} \varphi_{1}\right), \quad a_{12}=d\left(\varphi_{1}-\varphi_{2}\right) \\
a_{21}=d \lambda_{1} \lambda_{2}\left(\varphi_{2}-\varphi_{1}\right), \quad a_{22}=d\left(\lambda_{1} \varphi_{1}-\lambda_{2} \varphi_{2}\right) \\
d=\frac{1}{\lambda_{2}-\lambda_{1}}, \quad B=\frac{u_{s 2}-u_{r 2}}{\chi V}, \quad \varphi_{i}=\omega_{i} \cot \left(\omega_{i} \tau / 2\right) \quad(i=1,2)
\end{gathered}
$$

For the following values of the parameters:

$$
\begin{gathered}
\chi=0.4001, \quad \eta=4, \quad V=0.6773, \quad u_{s 1}=0.0127 \\
u_{s 2}=0.5, \quad B=-2.4994
\end{gathered}
$$

we obtain $\tau=2.65, T=2.2407$.

The corresponding behaviors of the two masses are shown on Figs. 2 and 3.

The constraints

$$
x_{1}^{\prime}-V<0(0<t<\tau)
$$

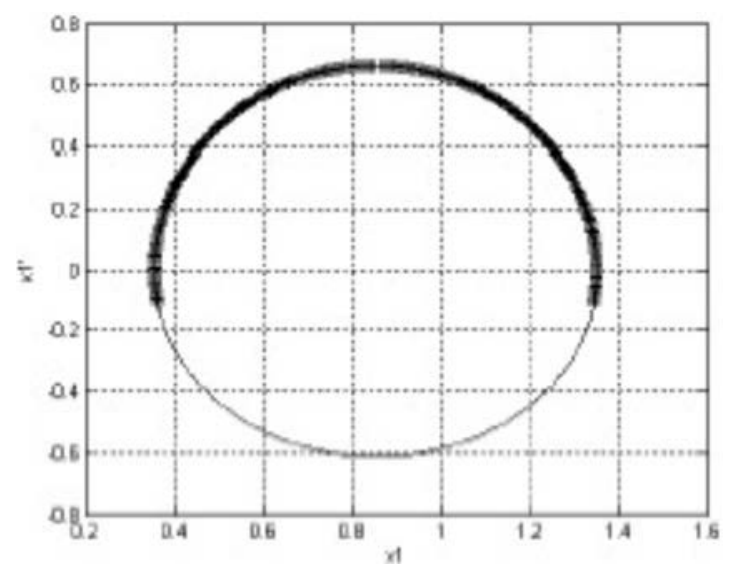

Fig. 2 Phase portrait of the slip-stick orbit (first mass)

$$
\begin{gathered}
z_{1}=\chi\left(x_{2}-x_{1}\right)-u_{r 2}<0 \\
z_{2}=\chi\left(x_{2}-x_{1}\right)+u_{r 2}>0, \quad(\tau<t<\tau+T)
\end{gathered}
$$

are checked on Figs. 4 and 5.

Solution II. For $\chi=0.5, \eta=1, V=1, u_{s 1}=u_{s 2}=1, u_{r 1}=3.4983$, we obtain $\tau=2, \tau_{1}=1.651, T=3.651, u_{r 2}=1.3569$.

The corresponding behavior of the system is shown on Figs. 6 and 7 .

The constraints

$$
p_{1} \equiv \chi\left(x_{2}-x_{1}\right)+u_{r 2}>0
$$

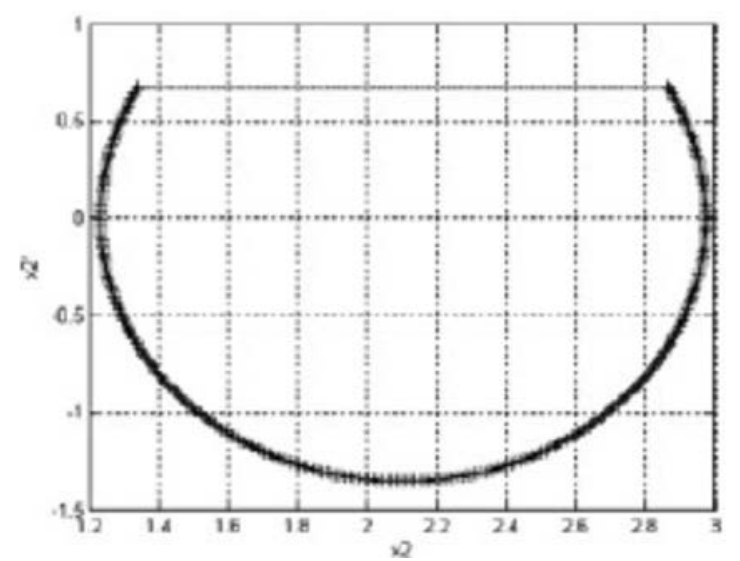

Fig. 3 Phase portrait of the slip-stick orbit (second mass)

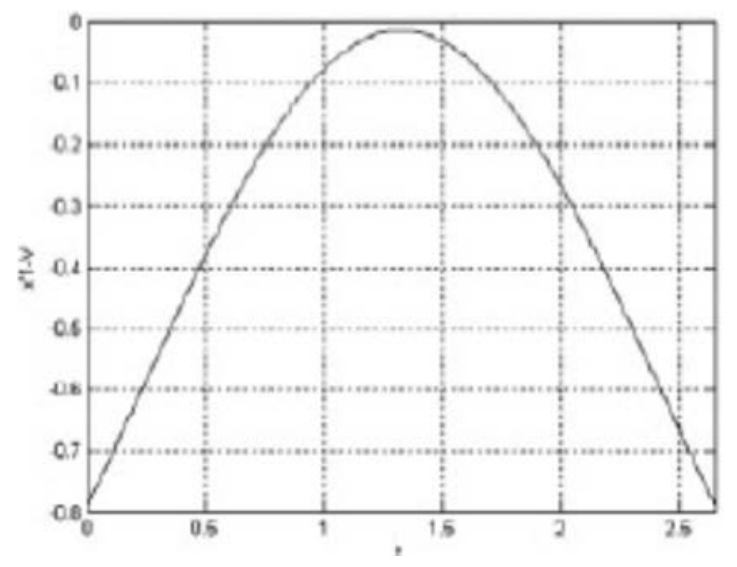

Fig. 4 Constraint $x_{1}^{\prime}-V<0$ 


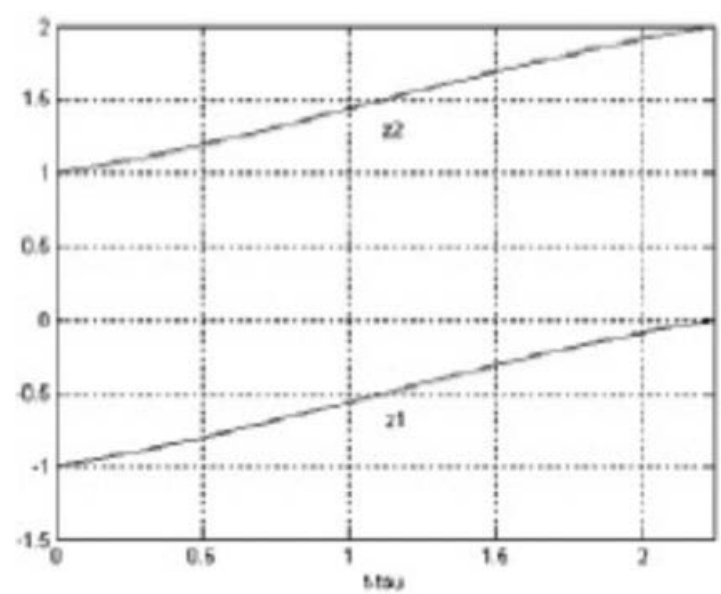

Fig. 5 Constraints $z_{1}<0, z_{2}>0$

$$
\begin{gathered}
p_{2} \equiv-\chi\left(x_{2}-x_{1}\right)+u_{r 2}>0, \quad(0<t<\tau) \\
q_{1} \equiv x_{1}-\chi x_{2}+u_{r 1}>0 \\
q_{2} \equiv-x_{1}+\chi x_{2}+u_{r 1}>0 \quad\left(\tau+\tau_{1}<t<\tau+\tau_{1}+T\right)
\end{gathered}
$$

are checked on Figs. 8 and 9.

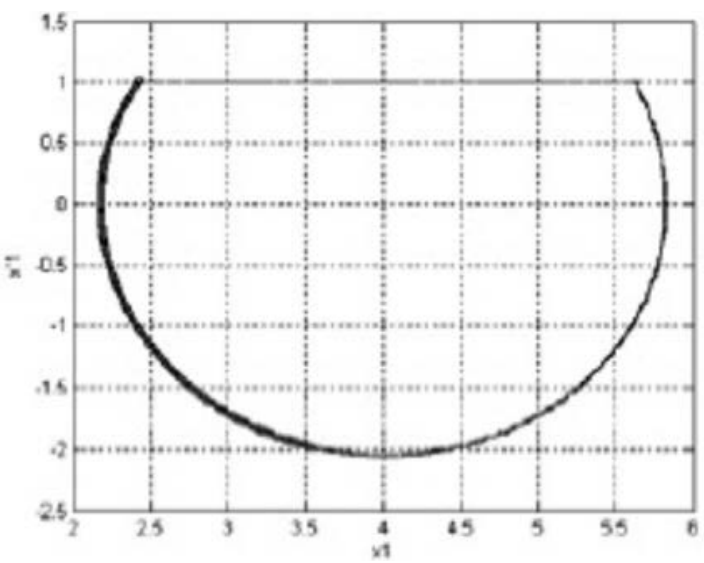

Fig. 6 Phase portrait of the first mass (Solution II)

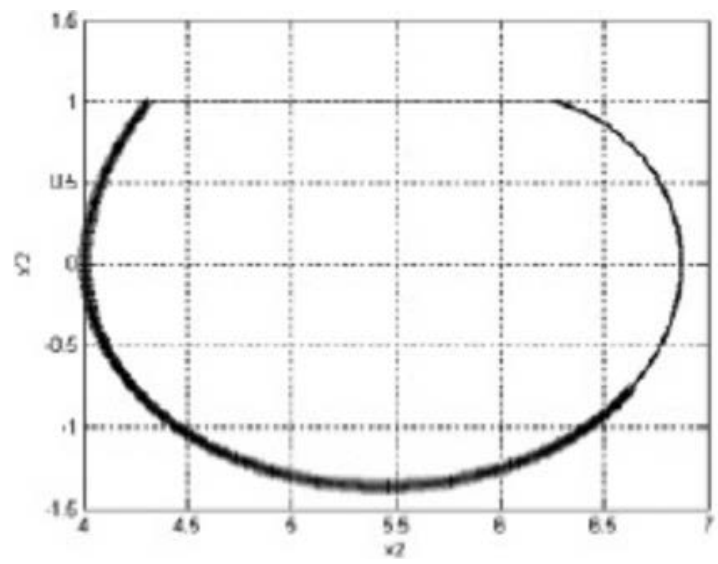

Fig. 7 Phase portrait of the second mass (Solution II)

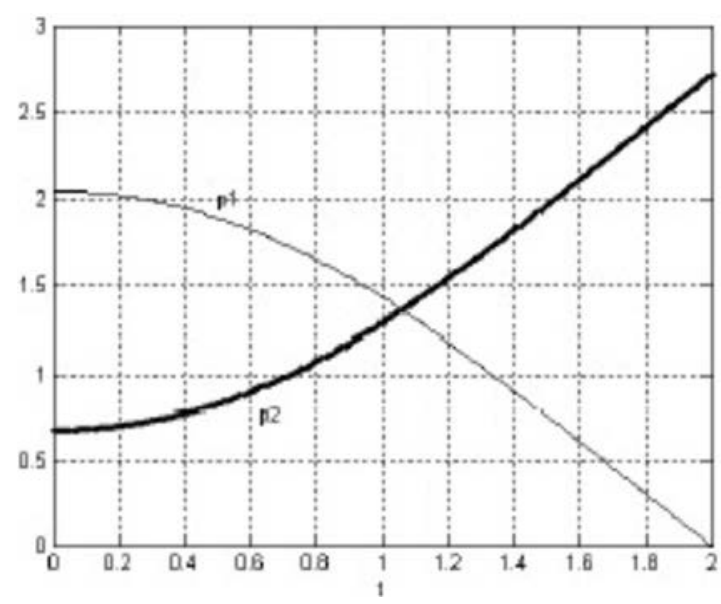

Fig. 8 Constraints $p_{1}>0, p_{2}>0$

\section{Conclusion}

Two kinds of periodic solutions for a two-degree of-freedom oscillator excited by dry friction have been obtained in analytical form. These two solutions differ by the number of switches occurring during one period. In Ref. [4], similar solutions have been obtained by numerical methods. However, in the work performed in Ref. [4], the friction model is not Coulomb's friction law.

The investigation of other kinds of periodic motions will be the subject of future researches, together with the analysis of the stability of the obtained periodic motions.

\section{Appendix: Symmetry of the Solution}

For $0<t<\tau / 2$,

$$
Z(\tau-t)=H(\tau-t) Z_{0}
$$

From the identies

$$
H(\tau-t)=H(-t) H(\tau), \quad Z(\tau)=H(\tau) Z_{0}=E Z_{0}, \quad H(-t) E=E H(t)
$$

it results to

$$
Z(\tau-t)=E H(t) Z_{0}=E Z(t)
$$

In particular, for $t=\tau / 2$, this relation gives

$$
Z(\tau / 2)=E Z(\tau / 2)
$$

Hence, the following relation holds:

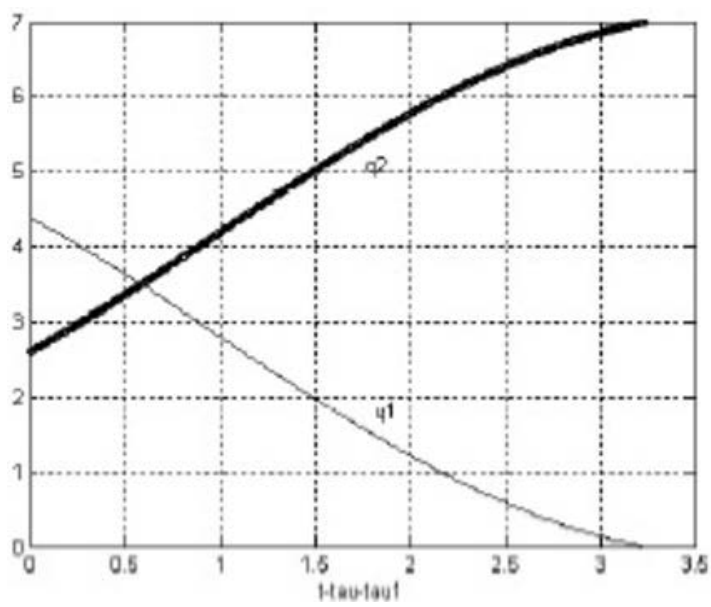

Fig. 9 Constraints $q_{1}>0, q_{2}>0$ 


$$
X(\tau / 2)=d_{0}
$$

A similar property of symmetry is obtained for the other part of the solution $(\tau<t<\tau+T)$. In particular, $X(\tau+T / 2)=d_{0}$.

\section{References}

[1] Awrejcewicz, J., and Delfs, J., 1990, "Dynamics of a Self-Excited Stick-Slip Oscillator With Two Degrees of Freedom, Part I. Investigation of Equilibria," Eur. J. Mech. A/Solids, 9(4), pp. 269-282.

[2] Liu, C. S., and Chang, W. T., 2002, "Frictional Behavior of a Belt-Driven and Periodically Excited Oscillator,” J. Sound Vib., 258(2), pp. 247-268.
[3] Andreaus, U., and Casini, P., 2001, "Dynamics of Friction Oscillators Excited by a Moving Base and/or Driving Force," J. Sound Vib., 245(4), pp. 685-699.

[4] Awrejcewicz, J., Dzyubak, J. and Grebogi, C., 2005, "Estimation of Chaotic and Regular (Stick-Slip and Slip-Slip) Oscillations Exhibited by Coupled Oscillators With Dry Friction," Nonlinear Dyn., 42, pp. 383-394.

[5] Pascal, M., 2006, "Dynamics and Stability of a Two Degrees of Freedom Oscillator With an Elastic Stop,” ASME J. Comput. Nonlinear Dyn., 1(1), pp. 94-102.

[6] Luo, A. C. J., and Gegg, B. C., 2006, "Stick and Non-Stick Periodic Motions in Periodically Forced Oscillators With Dry Friction," J. Sound Vib., 291(12), pp. 132-168

[7] Khizgiyaev, S. V., 2007, "Stick-Slip Self Oscillations of Mechanical System With Two Degrees of Freedom," PMM, 71(4), pp. 40-57. 